\title{
Early Surgery for Pulmonary Tuberculosis
}

\author{
A BILAL M SALIM MS NABI M MUSLIM M ZAMAN A. JAVAID Y KHAN M AHMED SAHMED \\ Department of Cardiothoracic Surgery, Postgraduate Medical Institute, Lady Reading Hospital, Peshawar \\ Correspondence to Dr. Aamir Bilal
}

\begin{abstract}
Objective: The purpose of our study was to analyze current indications for surgery in tuberculosis and evaluate the outcome of early surgical intervention. Place and duration of study: Department of Cardiothoracic Surgery, Postgraduate Medical Institute, Lady Reading Hospital, Peshawar from June 2000 to July 2004. Material \& methods: Total number of cases was 132; M: F 105: 27. Age range was 20 to 79 years. Mean age was 48.4 years. The indications for surgical intervention included 5 cases of pulmonary aspergillioma, 9 cases of pneumothorax; 3 cases of pulmonary nodes and masses without histological diagnosis, 15 cases bronchiectasis, 12 cases of massive hemoptysis and 82 cases of pleural empyema while six patients with multi drug-resistant tuberculosis required surgical intervention. Results: The techniques utilized included lobectomy in 45 cases, pleural drainage in 20 cases, segmented pulmonary resection in 32 cases, surgical procedures on the chest wall in 17 cases, pneumonectomy in 10 cases, decortication in 8 cases. In 22 cases two or more procedures were performed on the same patient. In 26(19.6\%) cases various complications were noted of which wound infection was the most frequent. There was a mortality rate of 3.3\% (4 cases). Conclusion: Surgical treatment is indicated for the complication of TB and management of MDR TB. Early surgery is beneficial in patients whose disease is st ill localized and who can tolerate resection surgery; of particular importance is a healthy opposite lung, on which the patient would be dependent during and immediately after surgery.

Key words: Early surgery, tuberculosis
\end{abstract}

Surgery in pulmonary tuberculosis (TB) has gone through several phases in its history, from being the treatment of choice in earlier times, before the discovery of antituberculin drugs, to its current status as a treatment modality used only in special circumstances ${ }^{1,2,3}$. There are several important controversies surrounding the role of surgery in TB, which the present study will attempt to address in some depth.

The discovery of Mycobacterium tuberculosis in 1882 coincided with the introduction of surgical treatment by "extrapleural pneumothorax", as the procedure was called by Forlanini. For many years collapse of the lung was the principal treatment for $\mathrm{TB}$, with the principal aim of impeding the spread of the microorganism, which needs an aerobic medium for its growth. Thirty years later, Jacobeus described thoracoscopy for the resection of pleural adherences in $\mathrm{TB}^{4}$.

The discovery of streptomycin in 1943 began a new era in the treatment of this disease ${ }^{4}$. Since then, new drugs have been filling the therapeutic arsenal, relegating the role of surgery to second place. There remain, however, several clinical situations that can be treated surgically, and these occasionally provoke controversy. It seems clear that this therapeutic option is indicated in the management of some sequelae and complications of the disease, although the role that surgery could take in the treatment of multidrugresistant TB is more controversial. While some authors defend it as an alternative therapy ${ }^{5}$, others relegate it to special cases only ${ }^{6}$. The aim of the present study was to evaluate the results of early surgical intervention in patients who had contralateral lung free of disease and had good respiratory reserve.

\section{Material and methods:}

The patients included in the study were treated for TB symptoms regarded as indications for surgery in the Thoracic Surgery Department, PGMI, LRH. One hundred and thirty-two patients were included ( 105 males a nd 27 females), ranging in age from 20 to 79 (average age 48.4) (Table I). All of the patients who had good pulmonary function studies and healthy opposite lung w ere included regardless of the sputum status where as patients with diffuse disease involving the opposite lung were excluded from the study. In all of these c ases there were e volving complications in previously-diagnosed TB that required surgery, or TB was discovered during surgery. A preoperative examination was done in all patients, including computed tomography (CT). Pulmonary function tests were also performed, in addition to routine investigations.

Clinical and microbiological controls were done at 1 , 2,4 , and 6 months after surgical treatment. The surgical indications are summarized in Table 1 . The majority were elective ( 99 cases, $75 \%$ ). The procedures utilized were determined either by the pathology or by the diagnostic and therapeutic goals of the operation. Pulmonary resection and thoracotomy were d one with d ouble lumen tubes and carried out by posterolateral thoracotomy and standard operating techniques, covering the bronchial stump with intercostal muscle or parietal pleura.

All of the samples were studied microbiologically and histologically. In pulmonary neoplasms, medinastinal staging was performed in the same manner as in cases of bronchogenic carcinoma. All of the examples were studied microbiologically and histologically. The patients were 
observed closely during the postoperative period. The observation period lasted from 6 months to 1 year.

\section{Results:}

The indications for surgical treatment in our series are shown in Table II. The total number of procedures carried out is summarized in Table III. Particularly numerous are pulmonary resections to remove nodes, masses, aspergillomas, bronchiectasis and destroyed lungs or hemoptysis. Six patients had MDR TB. Thoracotomy was indicated in 9 cases because of pneumothorax, and in 82 cases due to pleural empyema which had failed to resolve with drainage. In 3 cases, thoracotomy was first performed through a residual pleural space (tuberculous pleural sequelae), a nd a nother was performed later to close with myoplasty. One year after surgery $110(90.1 \%)$ patients showed no signs of disease or residual symptoms. Postoperative mortalities (death within 30 days after surgery) were $4(3.3 \%)$. These included one patient with hemoptysis \& borderline lung function who had undergone pulmonary resection for aspergilloma and could not come off ventilator. In two cases death was a consequence of evolving complications associated with massive and persistent air leakage due to bronchopleural fistulae requiring surgical closure. One mortality occurred in a patient with massive air leakage and severe psychiatric deterioration, whose disease was complicated by widespread infection.

Postoperative complications appeared in 26(19.6\%) patients (Table IV). Wound infection occurred in 11, persistent a ir leak in 11 and residual a irspace in $3 \mathrm{c}$ ases. Out of 11 wound infection, 9 were in previous intubation sites and not the thoracotomy wound.

Table 1

\begin{tabular}{ll}
\hline Total number & 132 \\
Male & 105 \\
Female & 27 \\
Age range & $20-79$ years \\
Mean age & 48.4 years \\
\hline
\end{tabular}

Table II: Indication

\begin{tabular}{ll}
\hline Indication & $\mathrm{n}=132$ \\
\hline Aspergilloma & 05 \\
Pneumothorax & 09 \\
Unexplained Mass & 03 \\
Bronchiectasis & 15 \\
Massive Hemoptysis & 12 \\
Empyema & 82 \\
MDR TB & 06 \\
\hline
\end{tabular}

Table III: Procedure

\begin{tabular}{ll}
\hline Procedure & $\mathrm{n}=132$ \\
\hline Lobectomy & 45 \\
Pleural drainage & 20 \\
Segmental resection & 32 \\
Pneumonectomy & 10 \\
Chest wall & 17 \\
Decortication & 08 \\
\hline
\end{tabular}

Table IV: Morbidity \& mortality

\begin{tabular}{ll}
\hline & $\mathrm{n}=132$ \\
\hline Morbidity & $25 / 132(19.6 \%)$ \\
Wound infection & 11 \\
Air leak & 11 \\
Wound dehiscence & 01 \\
Residual space & 02 \\
Mortality & $04 / 132(3.3 \%)$ \\
\hline
\end{tabular}

\section{Discussion:}

The incidence of pulmonary tuberculosis has risen since 1990 and in some countries the resistant forms are becoming more and more frequent. Surgical treatment is once again needed to manage these problems. The surgical indications for $\mathrm{TB}$ are still a matter of discussion. Controversies continue over indications for surgery in some types of complications, and also over the right timing for surgery. In each case the solution varies according to the degree of complication and the risk to the patient ${ }^{2}$. It is also important to keep in mind the diseases that may occur simultaneously, as in our study, where we found a $71.2 \%$ rate of c o-morbidity, e specially c hronic diseases, su ch a s diabetes.

One of the most controversial aspects of TB surgery is the surgical treatment of multi drug resistant. The surgical treatment of this type of TB has been described with acceptable results, which has induced several authors ${ }^{7,9}$ to recommend excision of the lesion even before 3 months of medical treatment has proven ineffective. We have found in accordance with the others $3,5,11,12$ that early surgery under such condition has favourable outcome, regardless of sputum status at the time of surgery. Therefore early surgery eliminates the possibility of cross contamination of healthy lung.

The possibility of a new worldwide epidemic of drug resistant Mycobacterium tuberculosis is of concern ${ }^{3,14}$. In setting of localized disease, persistent sputum positivity or patient intolerance of medical therapy, pulmonary resection should be under taken ${ }^{15}$. Bronchiectasis as an evolving complication requiring surgery has decreased over the past few years ${ }^{16,17,18}$. This decrease is related to the improvement in medical treatment of TB. It still continues to be an indication for surgery, taking into account the limitations of the disease and good operating conditions $^{20,21}$. The evaluation of this kind of complication demands good imaging technology. We used high resolution CT thorax in such cases for detailed delineation of disease.

Surgery of destroyed lung presents a challenge to the surgeon because of the high rate of complications, particularly after pneumonectomy ${ }^{22,23}$. We performed 10 pneumonectomies and faced this type of complication twice. Dissection proximal to the bronchial stump should be kept to a minimum, in order to preserve intact as much as possible of its blood supply. It must also be covered by intercostal muscle, parietal pleura or mediastinal fat. Positive sputum, empyema and pneumonectomy on the 
right side constitute risk factors for postoperative morbidity ${ }^{24,25}$. The risk is also high when aspergilla infection coexists with the disease ${ }^{22}$.

The absence of a specific diagnosis of a pulmonary mass or node may warrant invasive procedures to rule out the presence of a neoplasm. The association between neoplastic disease and TB is well-known, and takes the form of scar cancer, which develops over tuberculus scars. One characteristic that arouses suspicion is the absence of improvement during tuberculostatic treatment. In this type of neoplasm, most often adenocarcinomas, a high incidence of stage I cancer has been reported, with a more favorable prognosis?. Our experience would tend to confirm these reports, since of the seven cases of neoplasm of the lung that we have seen, 5 were in stage I, 1 in stage II and 1 in stage IIIa.

The special tropism characteristic of destructive tubercular lesions for the setting of aspergillomas is wellknown. Resection anticipatory of evolving complications has been described. The treatment of asymptomatic persistent pulmonary cavities has been the subject of controversy. Some authors advocate resection for the prevention of aspergillomas. In our opinion, prophylactic excision is not justified if there is no other motive for surgery, such as recurring hemoptysis, or suspicion of neoplasm or aspergilloma.

The management of pneumothorax secondary to TB follows the classic guidelines for secondary pneumothorax. The first response should be to place a pleural drainage tube. If it fails because the pulmonary parenchyma does not expand or because of persistent air leaks, thoracotomy may be indicated; h owever, g eneral c onditions sh ould be kept in mind, and less aggressive treatment is preferred $^{28,29}$. Therefore when indicated surgery should not be delayed. With one lung ventilation and meticulous surgical techniques; bronchopleural fistula and other complications of TB can be dealt effectively ${ }^{30,2}$. Pleural reactivation of $\mathrm{TB}$ has been a threat in patients who did not receive major anti tuberculous drug therapy. Moreover chronic empyema is frequently complicated by bronchopleural fistula leading to a SO called mixed empyema characterized by a contamination with both $\mathrm{M}$. tuberculosis and common pyogens ${ }^{4,18,25}$ chronic empyema following TB adequately treated with antituberculous medication is seldom associated reactivation of TB in the pleural space. The diagnosis is easy in the patients with known sequelae of TB. Abundant sputum suggests broncho pleural fistula. Purulent fluid aspirated should be sent routinely for cultures as soon as empyema is confirmed adequate drainage should be instituted ${ }^{2,8,10}$. Once the patient has not responded to ATT and tube thoracostomy for drainage of pleural collection alone; the patient should be referred to a thoracic surgical unit for early surgical intervention to prevent development of fibrothorax.

\section{Conclusion:}

Surgery is generally indicated for complications of TB and isolated MDR TB. Our series of 132 patients with a mortality of $3.3 \%$, is reflective of the high prevalence of complicated TB locally, as well as good results of surgical intervention. Early surgery is recommended to prevent further progression of pulmonary lesion. Moreover, cross contamination of healthy lung can be prevented in cases of MDB TB, by early surgical intervention. The main factor determining surgical fitness, apart from general factors, is the condition of the opposite lung. If the opposite lung is healthy \& untouched by disease early surgical intervention, regardless of duration of ATT and sputum status is advocated. If the opposite lung too is diseased then surgery should be delayed till at least sputum becomes negative, and other factors such as PFT's are at acceptable levels.

\section{References:}

1. Pomerantz M, Scannell JG, Ginsberg RJ: Pulmonary Tuberculosis. In Pearson FG (ed.): Thoracic Surgery. Churchill Livingstone. New York, 1995; 470-476

2. Freixinet J: Surgical indications for treatment of Pulmonary Tuberculosis. World J Surg, 1997; 21: 475-479

3. Perelman MI, Strelzov VP: Surgery for Pulmonary Tuberculosis. World J Surg, 1997; 21: 457-467

4. Naef AP: The 1900 Tuberculosis epidemic-starting point of modern Thoracic Surgery. Ann Thorac Surg, 1993; 55: 1375-1378

5. Iseman $\mathrm{MD}$ : T reatment of multidrug-resistant tuberculosis. N Engl J Med, 1993; 329: 784-791

6. Joint Tuberculosis Committee of the British Thoracic Society: Control and prevention of tuberculosis in the United Kingdom: code of practice 1994. Thorax, 1994; 49: 1193-1200

7. Mouroux J, Maalouf J, Padovani B et al: Surgical management of pleuropulmonary tuberculosis. J Thorac Cardiovasc Surg, 1996;111: 662-670

8. Yim AP: The role of video-assisted thoracoscopic surgery in the management of pulmonary tuberculosis. Chest, 1996; 110: $829-832$

9. Rizzi A, Rocco G, Robustellini M et al: Results of surgical management of tuberculosis. Experience in 206 patients undergoing operation. Ann Thorac Surg, 1995; 59: 896-900

10. Ramakantan R, Bandekar VG, Gandhi MS et al: Massive hemoptysis due to pulmonary tuberculosis: control with bronchial artery embolization. Radiology, 1996; 200: 691694

11. Van Leuven M, De Groot M, Shean KP et al: Pulmonary resection as an adjunct in the treatment of multiple drugresistant tuberculosis. Ann Thorac Surg, 1997; 63: 13681372

12. Kir A, Tahaoglu K, Okur E, Hatipoglu T: Role of surgery in multidrug- resistant tuberculosis: results of 27 cases. Eur J Cardiothorac Surg, 1997; 12: 531-534

13. Pomeranz M, Brown JM: Surgery in the treatment of multidrug-resistant tuberculosis. Clin Chest Med, 1997; 18: 123-130

14. Pomerantz M, Madsen L, Goble M, Iseman M: Surgical management of resistant mycobacterial tuberculosis and 
other mycobacterial pulmonary infections. Ann Thorac Surg, 1991, 52: 1108-1112

15. Ono N, Satoh K, Yokomise $H$ et al: Surgical management of Mycobacterium avium complex disease. Thorac Cardiovasc Surg, 1997; 45: 311-313

16. Nelson KG, Griffith DE, Brown BA, Wallace RJ: Results of operation in Mycobacterium avium-intracellulare. Ann Thorac Surg, 1998; 66: 325-330

17. Shiraishi Y, Fukushima K, Komatsu H, Kurashima A: Early pulmonary resection for localized Mycobacterium avium complex disease. Ann Thorac Surg, 1998; 66: 183-186

18. Annest LS, Kratz JM, Crawford FA: Current results of treatment of bronchiectasis. J Thorac Cardiovasc Surg, 1982; 83: 546-550

19. Pomeranz M, Denton JR, Huitt GA et al: Resection of the right middle lobe and lingula for mycobacterial infection. Ann Thorac Surg, 1996; 62: 990-993

20. Hsu HS, Hsu WH, Huang BS, Huang MH: Surgical treatment of endobronchial tuberculosis. Scand Cardiovasc J, 1997; 31: 79-82

21. Watanabe $Y$, Murakami S, Oda $M$ et al: Treatment of bronchial stricture due to endobronchial tuberculosis. World J Surg, 1997; 21: 480-487

22. Freixinet J, Varela A, López L et al: Surgical treatment of childhood mediastinal tuberculous lymphadenitis. Ann Thorac Surg, 1995; 59: 644-646
23. Massard G, Dabbagh A, Wihlm JM et al: Pneumonectomy for chronic infection is a h igh-risk procedure. A nn Thorac Surg, 1996; 62: 1033-1037

24. Halezeroglu S, Keles M, Uysal A et al: Factors affecting postoperative morbidity and mortality in destroyed lung. Ann Thorac Surg, 1997; 64: 1635-1638

25. Reed CE: Pneumonectomy for chronic infection: fraught with danger? Ann Thorac Surg, 1995; 59: 408-411

26. Battaglini JW, Murray GF, Keagy BA et al: Surgical management of symptomatic pulmonary aspergilloma. Ann Thorac Surg, 1985; 39: 512-516

27. Chen JC, Chang YL, Luh SP et al: Surgical treatment for pulmonary aspergilloma: a 28 year experience. Thorax, 1997; 52: 810-813

28. Massard G, Thomas P, Barsotti P et al: Long-term complications of extraperiosteal plombage. Ann Thorac Surg, 1997; 64: 220-225

29. Jouveshomme S, D autzenberg B, B akdach H, D erenne JP: Preliminary results of collapse therapy with plombage for pulmonary diseasecaused by multidrug-resistant mycobacteria. Am J Respir Crit Care Med, 1998; 157: 1609. 1615

30. Shiraishiy, Fukusshima Komatsu. Early pulmonary resection for localized Mycobacterium avium complex disease. Ann Thorac Surg 1998; 66(1): 183 -6. 